\title{
Avaliação das propriedades tecnológicas das madeiras maranhenses voltadas para a fabricação de violões
}

\section{Evaluation of technological properties of wood aimed Maranhão to manufacture guitars}

\author{
David Guilhon, Universidade Ceuma \\ david.guilhon@ceuma.br
}

\author{
Karoline de Lourdes Monteiro Guimaraes, Universidade Ceuma \\ karoline.guimaraes@ceuma.br
}

\author{
Anna Karen Lima Lourenço, Universidade Ceuma \\ annakaren16@gmail.com
}

\section{Resumo}

Instrumentos musicais fazem parte da vida humana desde tempos remotos, tornando-se até hoje importantes e usados para os diversos fins. O processo produtivo por trás de um violão evidencia a madeira como principal material, por conta de suas características únicas, mas escassas, caras e inviabilizando o fabrico. Este estudo pretende comparar espécies maranhenses com madeiras mundialmente usadas, usando propriedades tecnológicas como referências. Geraram-se, via Método de Wegst (densidade $\times$ elasticidade), gráficos de dispersão para cada uma das 5 partes do instrumento que, juntamente com as demais grandezas (módulo de ruptura à flexão, dureza Janka e coeficiente de anisotropia) e trabalhabilidade. Assim, 9 das 23 madeiras locais apresentaram-se como alternativas satisfatórias para tampos, fundos e laterais, braços, escalas e cavaletes de violão.

Palavras-chave: Madeiras maranhenses, Violão, Design.

\begin{abstract}
Musical instruments have been present in human life since ancient times, becoming important today and used for various purposes. The production chain behind a guitar shows wood as the main material, due to its unique characteristics, but scarce, expensive, making manufacturing impossible. This work aims to compare species from Maranhão with wood used worldwide, with technological properties as references. Scatter plots for each of the 5 parts of the instrument were generated using the Wegst Method (density $\times$ elasticity), which, together with the other quantities (flexural rupture module, Janka hardness and anisotropy coefficient) and workability. Therefore, 9 of the 23 native woods presented themselves as satisfactory alternatives for tops, backs and sides, necks, fretboards and guitar bridge.
\end{abstract}

Keywords: Woods from Maranhao, acoustic guitar, design 


\section{Introdução}

Desde tempos remotos, o ato de fabricar instrumentos musicais acompanha o homem em sua vida. Andrade (2015) traz a descoberta da música como um marco divisor entre povos primitivos e civilizações antigas. Por não haver entre os primeiros evidências científicas de sua existência, bem como falta de consciência de sua produção; coisas que já são observadas com certa sistematização nas primeiras civilizações. Como qualquer artefato, os instrumentos musicais sofreram evolução, onde no primeiro momento apresentavam-se com ruídos e sons não refinados, até chegar aos instrumentos modernos (ANDRADE, 2015). Tratando-se do cenário atual, os instrumentos musicais mostram-se, como quer Chiarelli (2005), como objetos desejados pelas pessoas para diversos fins (profissionais, lazer, hobbie, coleção, desenvolvimento pessoal, terapia).

Para que estes objetos sejam utilizados, há um processo produtivo que envolve a luteria. Alves, Longui e Armano (2008) consideram tal atividade como a fusão da arte com a tecnologia, observando a forte presença humana em meio a maquinários e equipamentos mecânicos. Essa mão humana, em convergência com tecnologias, vivências e percepções pessoais do luthier, também se mistura à ecologia da madeira, conceitos, machados e serras (CLAYTON; HERBERT; MIDDLETON, 2003).

Fruto de toda essa criativa mistura, o violão é considerado um dos instrumentos mais populares do mundo (BENNETT, 2016). Dessa forma, Brémaud (2012) frisa que a escolha dos materiais que o constituem possui papel importantíssimo para a projetação e fabricação, além de influenciar diretamente no cenário musical e identidade cultural do local onde é fabricado. Por estes motivos é que a madeira continua sendo o material preferido para a confecção de instrumentos musicais de cordas, mesmo com o crescimento avanço de pesquisa de novos materiais (WEGST, 2006; YOSHIKAWA; SHINODUKA; SENDA, 2008; YOSHIKAWA; WALTHAM, 2014; BENNETT, 2016; NCUBE; MASILINGA, 2017), suas propriedades tecnológicas são parâmetros cruciais para tal (SLOOTEN; SOUZA, 1993; WEGST, 2006; BUCUR, 2006; ARAMAKI; BRANCHERIAU; YSTAD, 2007; BRÉMAUD et al., 2008; LONGUI; LOMBARDI; ALVES, 2011; YOSHIKAWA; WALTHAM, 2014), pois estas características possuem ligação direta com as propriedades acústicas.

Mesmo com tantos pontos favoráveis, existem as delimitações que justificam a ocorrência de estudos sobre madeiras alternativas para a fabricação de violões mundo a fora. A inviabilidade, em termos econômicos e ambientais, da coleta de certas espécies) em decorrência de sua pouca quantidade) é, para Bennett (2016), um problema. Sproßmann, Zauer e Wagenführ (2017) dão como exemplo o caso do Jacarandá como um processo produtivo não sustentável, por conta do longo tempo em que a madeira demora para atingir as características desejadas. Isso acaba culminando na escassez de outras espécies, tornando-se o motivo de preocupação de pesquisadores e até alguns fabricantes. Tal fato resulta em pesquisas de espécies alternativas e/ou nativas para a manufatura de violões e outros instrumentos cordofones, em nível nacional (LONGUI; 2009; PORTELA, 2014; FLORES, 2015; COSTA, 2017) e internacional (BESSA, 2000; WEGST, 2006; YOSHIKAWA; SHINODUKA; SENDA, 2008; TELES, 2016; NCUBE; MASILINGA, 2017). 
O mesmo interesse também se replica particularmente na verificabilidade das espécies nativas do Maranhão, já que a sua flora possui grande disponibilidade de espécies, que podem ser aproveitadas comercialmente para vários setores, conforme o estudo de Vasconcellos et al. (2001). Tanto que este estudo é fruto de um projeto de Iniciação Científica do curso de Design, da Universidade Ceuma.

Diante do que foi escrito, o objetivo desta pesquisa é de análise comparativa entre espécies comercializadas em solo maranhense e madeiras tradicionalmente utilizadas para a fabricação de violões, baseadas em propriedades físico-mecânicas descritas na literatura, identificando a possibilidade de sua utilização nas diversas partes de um violão.

\section{Metodologia utilizada}

Esta pesquisa apresenta o estudo comparativo entre espécies tradicionalmente utilizadas (madeiras-referência) e madeiras nativas e comercializadas no Maranhão. As consideradas como tradicionais pautam-se nos estudos de Bennett (2016) e Ncube e Masilinga (2017), onde estão categorizadas por partes do instrumento, conforme compatibilidade de características da madeira com a referida peça. Adotou-se a nomenclatura descrita por Zaczéski et al. (2018) correspondem ao tampo (T), o fundo e as laterais (FL), o braço (B), a escala (E) e o cavalete (C). Já as madeiras locais estudadas fazem parte das espécies analisadas nos estudos de Nahuz et al. (2013) e Vasconcellos et al. (2001).

As características tecnológicas avaliadas seguem a literatura específica (WEGST, 2006; YOSHIKAWA; SHINODUKA; SENDA, 2008; KÚDELA; KUNŠTÁR, 2011; BENNETT, 2016, SPROßMANN et al., 2017). As propriedades que norteiam a análise em termos físicos são as contrações tangenciais (Tan), radiais (Rad) e volumétricas (Vol). Enquanto as características sob os aspectos mecânicos são a densidade $(\rho)$, módulo de elasticidade (E), módulo de ruptura à flexão $(G)$ e dureza Janka $(H j)$.

A partir dos dados destas propriedades, geraram-se 5 gráficos de dispersão (cada um referente a uma peça do instrumento) que cruzam densidade ( $\rho$ ) e módulo de elasticidade (E) como indicativo de qualidade acústica, auxiliados pelo espectro gerado pela elipse de confiança $95 \%$ resultante dos valores das madeiras-referência. Também pôde-se trabalhar com quatro parâmetros classificatórios, que são: semelhantes às padrão, próximas das padrão, dentro do espectro e fora do espectro. O módulo de ruptura à flexão $(\mathrm{G})$, dureza Janka $(\mathrm{Hj})$ e coeficiente de Anisotropia (CAnisot) (índice fruto da razão entre contrações tangencial e radial) ajudam na avaliação comparativa direta, valendo-se de taxas máximas e mínimas de cada de cada propriedade. A trabalhabilidade é, ainda, considerada como fator classificatório e eliminatório, observando nível de facilidade do processamento da madeira na fabricação, desembocando na viabilidade técnica. 


\section{Resultados e discussões}

Analisaram-se 23 espécies de madeiras maranhenses, sendo comparadas com 20 tipos de madeiras tradicionalmente usadas para a fabricação de violões, sendo que estas estão devidamente classificadas conforme a aplicação nas 5 partes do instrumento.

Tabela 1: Propriedades tecnológicas das madeiras tradicionalmente usadas. Fonte: Adaptado de Bennett (2016); Ncube e Masilinga (2017).

\begin{tabular}{|c|c|c|c|c|c|c|c|c|}
\hline \multicolumn{9}{|c|}{ PROPRIEDADES FÍSICO-MECÂNICAS DAS MADEIRAS TRADICIONALMENTE USADAS } \\
\hline \multirow{3}{*}{$\begin{array}{c}\text { PARTES } \\
\text { DO } \\
\text { VIOLÃO }\end{array}$} & \multirow{3}{*}{ ESPÉCIES } & \multicolumn{4}{|c|}{ FÍSICAS } & \multicolumn{3}{|c|}{ MECÂNICAS } \\
\hline & & \multicolumn{3}{|c|}{ Contração (\%) } & \multirow{2}{*}{$\boldsymbol{\rho}\left(\mathrm{kg} \mathrm{m}^{3}\right)$} & \multirow{2}{*}{$\mathbf{E}(\mathrm{GPa})$} & \multirow{2}{*}{$\begin{array}{c}\mathbf{G} \\
(\mathrm{MPa})\end{array}$} & \multirow{2}{*}{$\mathbf{H}_{\mathbf{J}}(\mathrm{N}$} \\
\hline & & Tag & Rad & Vol & & & & \\
\hline \multirow{5}{*}{ Tampo } & $\begin{array}{l}\text { Abeto da Noruega ou Pinho-alemão } \\
\text { (Piceas abies) }\end{array}$ & 8,2 & 3,9 & 12,9 & 405 & 9,7 & 63 & 1680 \\
\hline & Espruce-de-Sitka (Piceas sitchensis) & 4,3 & 7,5 & 11,5 & 425 & 11,03 & 70 & 2270 \\
\hline & $\begin{array}{l}\text { Abeto vermelho de Elgelmann (Piceas } \\
\text { engelmannii) }\end{array}$ & 3,8 & 7,1 & 11 & 385 & 9,44 & 62,2 & 1740 \\
\hline & Abeto de prata (Albies albes) & 4 & 8,7 & 12,8 & 415 & 8,28 & 66,1 & 1420 \\
\hline & Jacarandá do Brasil (Dalbergia nigra) & 2,9 & 4,6 & 8,5 & 835 & 13,93 & 135 & 12410 \\
\hline \multirow{7}{*}{$\begin{array}{l}\text { Fundo e } \\
\text { laterais }\end{array}$} & Jacarandá do Brasil (Dalbergia nigra) & 2,9 & 4,6 & 8,5 & 835 & 13,93 & 135 & 12410 \\
\hline & Jacarandá indiano (Dalbergia latifolia) & 2,7 & 5,9 & 8,5 & 830 & 11,5 & 114,4 & 10870 \\
\hline & $\begin{array}{l}\text { Mogno hondurenho (Swietenia } \\
\text { macrophylla) }\end{array}$ & 2,9 & 4,3 & 7,5 & 590 & 10,06 & 80,8 & 4020 \\
\hline & Mogno cubano (Swietenia mahogani) & 3 & 4,6 & 8 & 600 & 9,31 & 74,1 & 4120 \\
\hline & Acácia (Acacia koa) & 5,5 & 6,2 & 12,4 & 610 & 10,37 & 87 & 5180 \\
\hline & Cedro Espanhol (Cedrela odorata) & 4,1 & 6,2 & 10,2 & 470 & 9,12 & 70,8 & 2670 \\
\hline & Bordo-açucareiro (Acer saccharum) & 4,8 & 9,9 & 14,7 & 610 & 12,62 & 109 & 6450 \\
\hline \multirow{9}{*}{ Braço } & Jacarandá indiano (Dalbergia latifolia) & 2,7 & 5,9 & 8,5 & 830 & 11,5 & 114,4 & 10870 \\
\hline & $\begin{array}{c}\text { Mogno hondurenho (Swietenia } \\
\text { macrophylla) }\end{array}$ & 2,9 & 4,3 & 7,5 & 590 & 10,06 & 80,8 & 4020 \\
\hline & Acácia (Acacia koa) & 4,8 & 9,9 & 14,7 & 610 & 12,62 & 109 & 6450 \\
\hline & Mogno cubano (Swietenia mahogani) & 3 & 4,6 & 8 & 600 & 9,31 & 74,1 & 4120 \\
\hline & Cedro Espanhol (Cedrela odorata) & 4,1 & 6,2 & 10,2 & 470 & 9,12 & 70,8 & 2670 \\
\hline & Padauk africano (Pterocarpus soyauxii) & 3,3 & 5,2 & 7,6 & 745 & 11,72 & 116 & 8760 \\
\hline & Pau-roxo (Peltogyne spp) & 3,8 & 6,4 & 10,6 & 905 & 20,26 & 151,7 & 11190 \\
\hline & Nogueira-preta (Juglans nigra) & 5,5 & 7,8 & 12,8 & 610 & 11,59 & 100,7 & 4490 \\
\hline & Bubinga (Guibourtia demeusei) & 6 & 8,2 & 13,9 & 840 & 18,41 & 168,3 & 10720 \\
\hline \multirow{7}{*}{ Escala } & Jacarandá do Brasil (Dalbergia nigra) & 2,9 & 4,6 & 8,5 & 835 & 13,93 & 135 & 12410 \\
\hline & Jacarandá indiano (Dalbergia latifolia) & 2,7 & 5,9 & 8,5 & 830 & 11,5 & 114,4 & 10870 \\
\hline & Ébano do Ceilão (Diospyros ebenum) & 5,4 & 8,8 & 14,3 & 915 & 14,07 & 128,6 & 10790 \\
\hline & Bordo-açucareiro (Acer saccharum) & 4,8 & 9,9 & 14,7 & 610 & 12,62 & 109 & 6450 \\
\hline & Ébano africano (Diospyros crassiflora) & 8,3 & 11,2 & 19,6 & 955 & 16,89 & 158,1 & 13700 \\
\hline & Ébano Makassar (Diospyros celebica) & - & - & - & 1120 & 17,35 & 157,2 & 14140 \\
\hline & Bubinga (Guibourtia demeusei) & 6 & 8,2 & 13,9 & 840 & 18,41 & 168,3 & 10720 \\
\hline \multirow{3}{*}{ Cavalete } & Ébano africano (Diospyros crassiflora) & 8,3 & 11,2 & 19,6 & 955 & 16,89 & 158,1 & 13700 \\
\hline & Ébano Makassar (Diospyros celebica) & - & - & - & 1120 & 17,35 & 157,2 & 14140 \\
\hline & Ébano do Ceilão (Diospyros ebenum) & 5,4 & 8,8 & 14,3 & 915 & 14,07 & 128,6 & 10790 \\
\hline
\end{tabular}

A Tabela 1 apresenta estas madeiras-referência com suas características tecnológicas, conforme versa a literatura (BENNETT, 2016; NUCBE; MASILINGA, 2017), que são: Abeto da Noruega (PA), Espruce de Sitka (PS), Pinus Engelmann (PE), Abeto de prata (AA), Jacarandá do 
Brasil (DN), Jacarandá indiano (DL), Mogno brasileiro (SM), Mogno (SW), Acácia (AK), Cedro Espanhol (CO), Bordo-açucareiro (AS), Paudak africano (PT), Pau-roxo (PY), Nogueira-preta (JN), Bubinga (GD), Ébano do Ceilão (DE), Ébano Massakar (DY) e Ébano africano (DC).

Tabela 2: Propriedades tecnológicas das madeiras maranhenses. Fonte: Adaptado de Vasconcellos et al. (2001), Nahuz et al. (2013) e IPT (2018).

\section{PROPRIEDADES TECNOLÓGICAS DAS MADEIRAS MARANHENSES}

\begin{tabular}{|c|c|c|c|c|c|c|c|}
\hline \multirow{3}{*}{ ESPÉCIES } & \multicolumn{4}{|c|}{ FÍSICAS } & \multicolumn{3}{|c|}{ MECÂNICAS } \\
\hline & \multicolumn{3}{|c|}{ Contração (\%) } & \multirow[t]{2}{*}{$\begin{array}{c}\mathbf{\rho} \\
\left(\mathrm{kg} / \mathrm{m}^{3}\right)\end{array}$} & \multirow[t]{2}{*}{$\mathbf{E}(\mathrm{GPa})$} & \multirow[t]{2}{*}{$\begin{array}{c}\mathbf{G} \\
(\mathrm{MPa})\end{array}$} & \multirow[t]{2}{*}{$\mathbf{H j}(\mathrm{N})$} \\
\hline & Tag & Rad & Vol & & & & \\
\hline $\begin{array}{c}\text { Angelim } \\
\text { (Hymenolobium petraeum Ducke) }\end{array}$ & 6,3 & 4,1 & 10,1 & 590 & 11,572 & 109,3 & 5325 \\
\hline $\begin{array}{c}\text { Bacuri } \\
\text { (Platonia insignis Mart.) }\end{array}$ & 8,1 & 4,6 & 13,4 & 670 & 12,739 & 96,8 & 6953 \\
\hline $\begin{array}{c}\text { Cachaceiro } \\
\text { (Coupia glabra Aubl.) }\end{array}$ & 9,1 & 4,8 & 16,1 & 710 & 17,142 & 67,2 & 6266 \\
\hline $\begin{array}{c}\text { Cedro } \\
\text { (Cedrela odorata L.) }\end{array}$ & 6,2 & 4 & 11,6 & 440 & 8,336 & 81,2 & 3138 \\
\hline $\begin{array}{c}\text { Cedrorana } \\
\text { (Vochysia vismiaefolia Sp. Ex Warm.) }\end{array}$ & 8,8 & 4 & 12,1 & 490 & 11,18 & 91,2 & 5492 \\
\hline $\begin{array}{l}\text { Cerejeira-da-Amazônia } \\
\text { (Torresa acreana Ducke) }\end{array}$ & 6,2 & 2,9 & 9,3 & 600 & 9,277 & 88,5 & 3325 \\
\hline $\begin{array}{c}\text { Copaíba } \\
\text { (Copaifera multijuga Hayne) }\end{array}$ & 7,1 & 3,8 & 13,4 & 575 & 10,339 & 112,5 & 4109 \\
\hline $\begin{array}{c}\text { Cumaru } \\
\text { (Dipteryx odorata (Aubli.) Willd.) }\end{array}$ & 8,2 & 5,3 & 13,6 & 908 & 19,306 & 59,3 & 9787 \\
\hline $\begin{array}{c}\text { Curupixá } \\
\text { (Couratari } s p .)\end{array}$ & 6,6 & 4,2 & 10,9 & 500 & 10,591 & 88,8 & 5315 \\
\hline $\begin{array}{c}\text { Freijó } \\
\text { (Cordia goeldiana Hub.) }\end{array}$ & 6,7 & 3,2 & 9,1 & 480 & 11,101 & 93,7 & 3932 \\
\hline $\begin{array}{c}\text { Goiabão } \\
\text { (Planchonella pachycarpa Pires) }\end{array}$ & 11,2 & 6,2 & 16,5 & 730 & 12,847 & 155,5 & 8120 \\
\hline $\begin{array}{c}\text { Guanandi } \\
\text { (Agonandra brasiliensis Miers) }\end{array}$ & 10,5 & 5,2 & 17 & 620 & 8,32617 & 83,4 & - \\
\hline $\begin{array}{c}\text { Jatobá } \\
\text { (Hymenaea courbaril L.) }\end{array}$ & 7,2 & 3,1 & 10,7 & 800 & 14,837 & 131,6 & 11180 \\
\hline $\begin{array}{c}\text { Louro-cravo } \\
\text { (Ocotea cymbarum H.B.K.) }\end{array}$ & 8,1 & 3,9 & 13,6 & 660 & 11,866 & 52,9 & 4717 \\
\hline $\begin{array}{c}\text { Louro-rosa } \\
\text { (Nectandra rubra Mez) }\end{array}$ & 10 & 4 & 15,9 & 642 & 10,032 & 71,5 & 3076 \\
\hline $\begin{array}{c}\text { Maçaranduba } \\
\text { (Manikara huberu (Ducke) Stand) }\end{array}$ & 11 & 6,8 & 19 & 833 & 14,769 & 117 & 9611 \\
\hline $\begin{array}{c}\text { Maracatiara } \\
\text { (Astronium lecointei Ducke) }\end{array}$ & 6,3 & 3,3 & 11,2 & 810 & 12,3 & 101,6 & 7737 \\
\hline $\begin{array}{c}\text { Mogno } \\
\text { (Swietenia macrophylla King) }\end{array}$ & 4,5 & 3,2 & 8,6 & 520 & 9,11 & 90,6 & 4942 \\
\hline $\begin{array}{c}\text { Pau-d'arco } \\
\text { (Tabebuia serratifolia (Dahl) Nichols) }\end{array}$ & 5,9 & 4 & 10,9 & 840 & 15,298 & 148,5 & 10807 \\
\hline $\begin{array}{c}\text { Piqui } \\
\text { (Caryocar glabrum (Aubl.) Pers.) }\end{array}$ & 13 & 5,7 & 21,1 & 850 & 13,258 & 103,6 & 4874 \\
\hline $\begin{array}{c}\text { Piquiá } \\
\text { (Caryocar villosum (Aubl.) Pers.) }\end{array}$ & 9,2 & 5,5 & 16,7 & 930 & 14,111 & 145,7 & 5864 \\
\hline $\begin{array}{c}\text { Sucupira } \\
\text { (Diplotropis purpurea Benth.) }\end{array}$ & 8,4 & 5,6 & 15,1 & 780 & 16,06 & 147,6 & 9542 \\
\hline $\begin{array}{c}\text { Tatajuba } \\
\text { (Bagassa guianensis) }\end{array}$ & 7,1 & 5,5 & 11,4 & 683 & 17,304 & 54,4 & 7208 \\
\hline
\end{tabular}


De igual modo, a Tabela 2 lista as mesmas propriedades das madeiras regionais, seguindo as pesquisas de Vasconcellos et al. (2001) e Nahuz et al. (2013), que são: Angelim (AG), Bacuri (BA), Cachaceiro (CA), Cedro (CD), Cedrorana (CE), Cerejeira-da-Amazônia (CJ), Copaíba (CP), Cumaru (CM), Curupixá (CX), Freijó (FR), Goiabão (GO), Guanandi (GU), Jatobá (JA), Louro-cravo (LC), Louro-rosa (LR), Maçaranduba (MÇ), Maracatiara (MR), Mogno (MG), Paud'arco (PD), Piqui (PI), Piquiá (PQ), Sucupira (SU) e Tatajuba (TA).

A Figura 1 apresenta os valores mínimos e máximos de cada uma das 5 propriedades trabalhadas neste artigo, distribuídas em cada uma das 5 partes do violão, atribuídos às madeirasreferência.

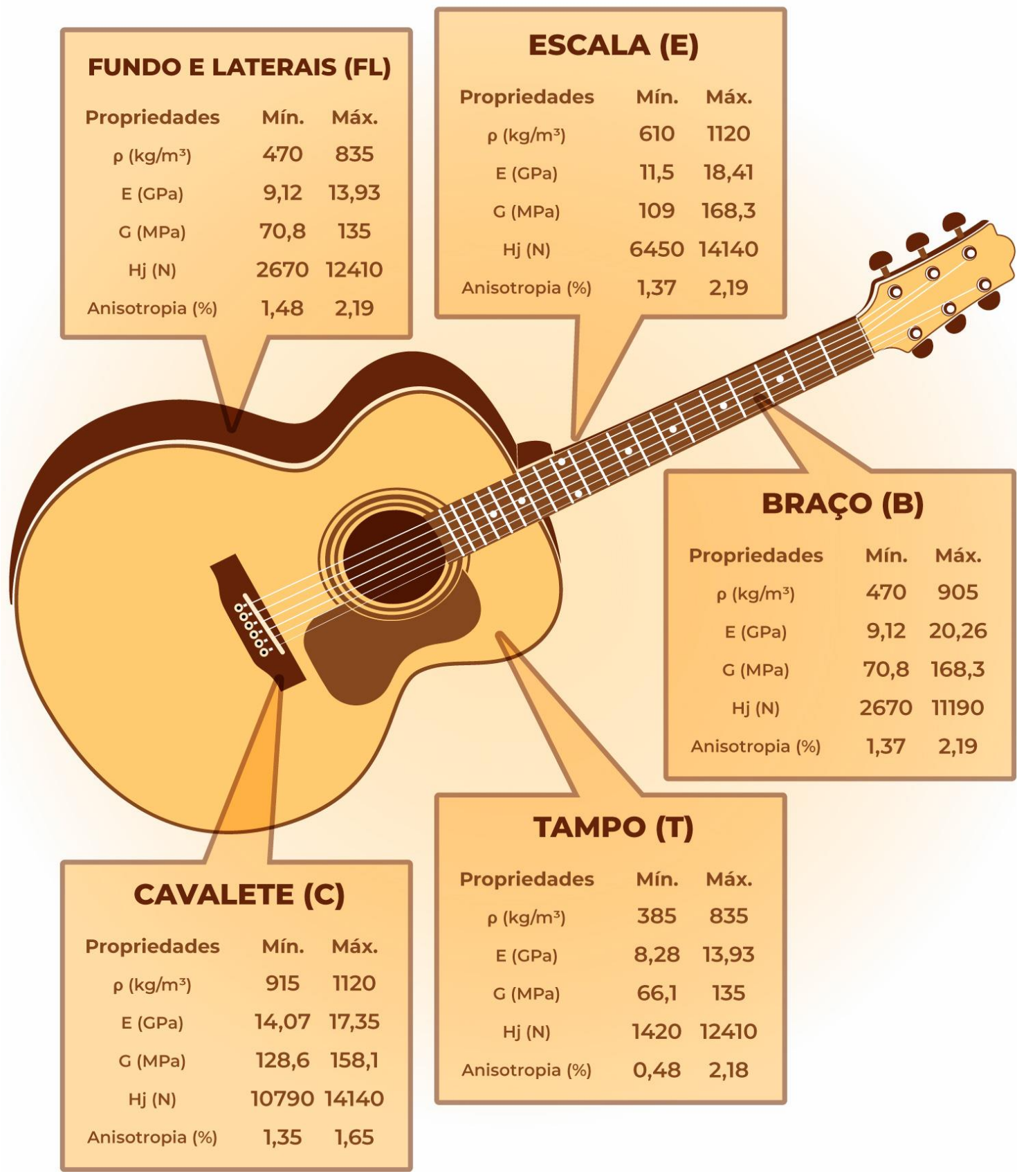

Figura 1: Violão e suas partes, com os valores mínimo e máximo das propriedades avaliadas. 
Observando-se as madeiras "tradicionais" e as maranhenses, verifica-se a ocorrência de duas espécies semelhantes: Cedrela odorata e Swietenia macrofilia. Cabe aqui apontar que para cada grupo seguiram-se os dados fornecidos pela literatura de cada realidade apontada. É interessante falar que tais espécies nativas, por conta da similaridade, serão aqui listadas e automaticamente classificadas como os exemplares mundialmente usados.

Em relação às propriedades tecnológicas, Wegst (2006) confrontou o módulo de elasticidade (E) e a densidade $(\rho)$ da madeira e mostrou que a relação entre essas propriedades indica quais madeiras podem ser usadas em instrumentos. O módulo de elasticidade relaciona-se à aplicação de uma força sobre o material e sua capacidade de voltar à forma original sem deformação, sendo uma propriedade importante para peças sujeitas a flexão ou compressão (OLIVEIRA; DELLA LUCIA; VITAL, 1988, p. 130), enquanto a densidade é importante por relacionar-se à resistência, uma vez que quanto mais densa, possivelmente será mais resistente. Soares (2014) complementa que madeiras menos densas tem maior tendência a absorção do som, enquanto as mais densas possuem propriedades vibracionais mais elevadas. Já Bennett (2016) ressalta que a ligação destas duas propriedades ( $\mathrm{E}$ e $\rho$ ) com características acústicas primárias (velocidade do som, impedância, coeficiente de radiação sonora e coeficiente de perda).

Outras propriedades também são observadas para a análise de madeiras, como o tipo de grã, trabalhabilidade e estabilidade dimensional. Portela (2014) e Flores (2015) trazem a preferência por madeiras com grã regular, cujas fibras não possuem desvios ou inclinações em relação ao eixo do tronco, por ser mais fácil para se conseguir um bom acabamento. Por sua vez, a estabilidade dimensional é pautada nos baixos valores de contração do material nos três eixos, onde Costa (2017) apresenta o coeficiente de anisotropia (razão entre a contração tangencial e a contração radial) como uma característica a ser considerada. Assim, quanto mais próximo de 1, menos defeitos por fendilhamentos e empenamentos a madeira terá. Moreschi (2009) indica como parâmetros para este índice a seguinte escala: 1,2 a 1,5 para madeiras consideradas excelentes (adequadas para instrumentos musicais); entre 1,6 e 1,9 para madeiras consideradas normais; e igual ou maior que 2,0 para madeiras de baixa qualidade, consideradas inaptas neste estudo.

Portanto, foram elaborados 5 gráficos de dispersão de cada uma das 5 partes analisadas do violão, tendo em eixos diferentes o módulo de elasticidade (E) e a densidade ( $\rho$ ). Os gráficos servirão como suporte argumentativo, onde os pontos azuis correspondem às madeiras-referência e os vermelhos às espécies locais.

\section{Tampo}

Gore (2011) afirma que o tampo é a principal superfície de irradiação do som o violão. Para tal peça, as madeiras ideais devem apresentar a combinação de alta rigidez com baixa densidade, pois estas ajudam a propagar o som com mais facilidade e mais volume (WEGST, 2006; GORE, 2011; BENNETT, 2016). Em concordância, Souza (1983) e Teles (2004) abordam que para uma espécie ser apta para o tampo, é necessário que tenha: grã direita, boa trabalhabilidade, boa estabilidade dimensional, facilidade na colagem e com bom acabamento. 
TAMPO (T)

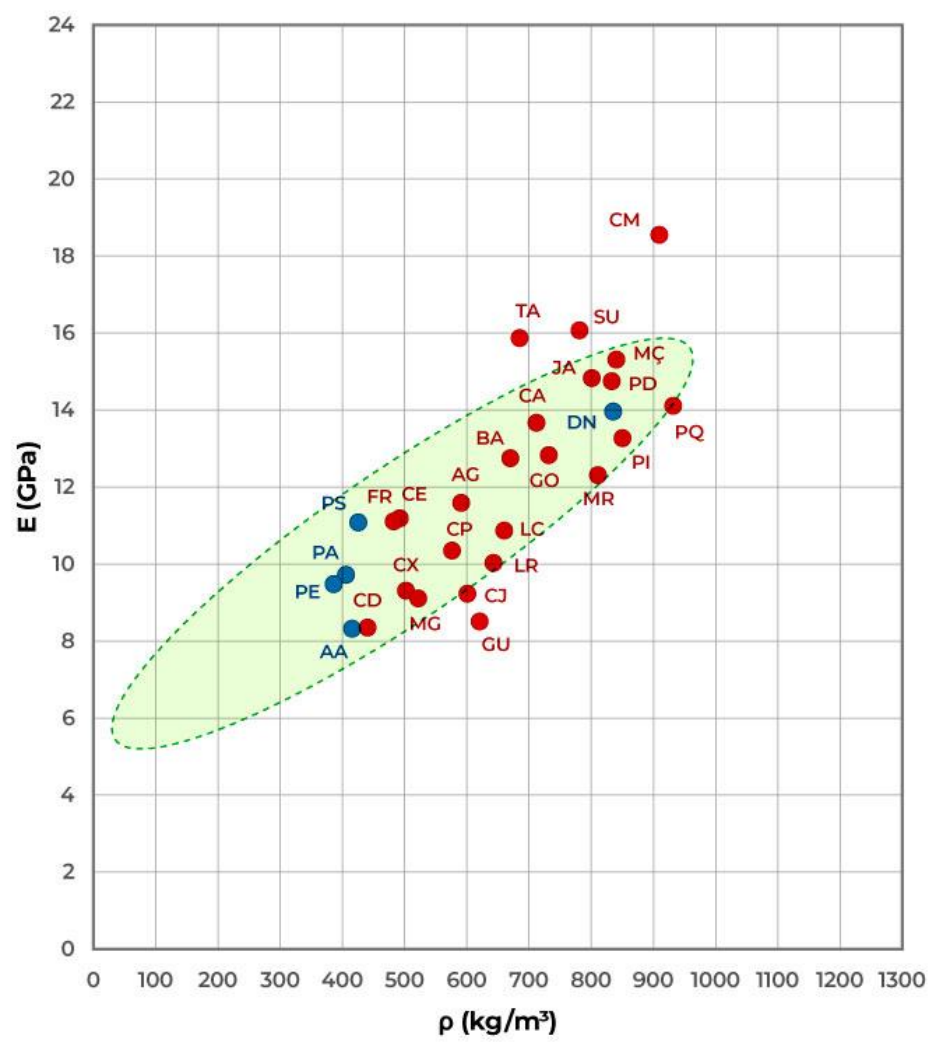

Figura 2: Gráfico de dispersão comparativo entre espécies tradicionalmente usadas e comercializadas no Maranhão para o tampo do violão. Onde: círculos azuis - PA=Piceas abies; PS=Piceas sitchensis; PE=Pinus engelmannii; $\mathbf{A A = A l b i e s ~ a l b e s ; ~}$

DA=Dalbergia nigra; círculos vermelhos - Angelim=AG; Bacuri=BA; Cachaceiro=CA; Cedro=CD; Cedrorana=CE;

Cerejeira-da-Amazônia=CJ; Copaíba=CP; Cumaru=CM; Curupixá=CX; Freijó=FR; Goiabão=GO; Guanandi=GU;

Jatobá=JA; Louro-cravo=LC; Louro-rosa=LR; Maçaranduba=MÇ; Maracatiara=MR; Mogno=MG; Pau-d'arco=PD; Piqui=PI; Piquiá=PQ; Sucupira=SU e Tatajuba=TA.

É possível observar na figura 2 que existem espécies nativas com valores aproximados das madeiras mundialmente usadas, como o Cedrorana, Curupixá e Freijó próximos a Piceas sitchensis. Apesar de um tanto mais distante dos abetos e espruces, a Dalbergia nigra tem o Jatobá, Pau-d'arco, Maçaranduba e Piquiá com taxas parecidas; vê-se o Piqui com taxa. Além destas espécies, encontram-se dentro desse espectro indicado pela elipse de confiança de 95\% delineada pelos valores das madeiras tradicionalmente usadas para o tampo: Angelim, Bacuri, Cerejeira-da-Amazônia, Copaíba, Goiabão, Louro-cravo, Louro-rosa e Maracatiara.

Em consonância com a fala de Souza (1983) e Teles (2004), apenas o Cedrorana, Curupixá e Freijó apresentam a grã direita e facilidade na trabalhabilidade. As madeiras perto do Jacarandá brasileiro oscilam entre estes dois fatores ou apresentam ambos desfavoráveis (grã inversa e/ou trabalhabilidade moderadamente difícil). Considerando-se grandezas como módulo de ruptura (G) e dureza Janka $(\mathrm{Hj})$, as madeiras nativas apresentaram valores acima dos números apresentados pelas madeiras-referência, aproximadamente 4 vezes maior. As nativas apresentaram de 3076 a 10807 N, já as referência ficaram entre 1420 e 2270 N, com exceção da Dalbergia nigra, que apresentou 12410 N. Em outras palavras, as nativas são mais duras que as "tradicionais". Ainda assim, todas as madeiras analisadas encontram-se dentro do espectro com relação a $E$ e $\rho$. Tal rigidez favorece a fabricação de tampos que proporcionam maior volume 
acústico sem muito esforço para a execução por parte do instrumentista, o que contribui para a qualidade para o violão a ser fabricado.

Porém, por serem fáceis de trabalhar, de grã direita e coeficiente de anisotropia abaixo de 2,0, as madeiras indicadas para o tampo são: Bacuri, Cedro, Curupixá e Goiabão.

Fundo e laterais

O fundo e as laterais funcionam como uma estrutura que recebe a vibração das cordas vindas do tampo, formando uma cavidade para a ressonância do ar, parte onde o efeito é irradiado pela boca do violão (WEGST, 2006; YOSHIKAWA; SHINODUKA; SENDA, 2008). A esse pensamento Gore (2011) complementa que a madeira do fundo funciona como uma superfície vibratória secundária que amplifica a tonalidade do tampo. Portanto, tal peça deve estar alinhada na mesma frequência do tampo. Já as madeiras para laterais devem ser flexíveis ao calor (NCUBE; MASILINGA, 2017; ZACZÉSKI et al., 2018), não podendo ser muito pesadas, possuir boa trabalhabilidade (colagem e acabamento) e ainda apresentar boa estabilidade dimensional. Por fim, não podem ter restrições quanto às propriedades mecânicas (SOUZA, 1983).

FUNDO E LATERAIS (FL)

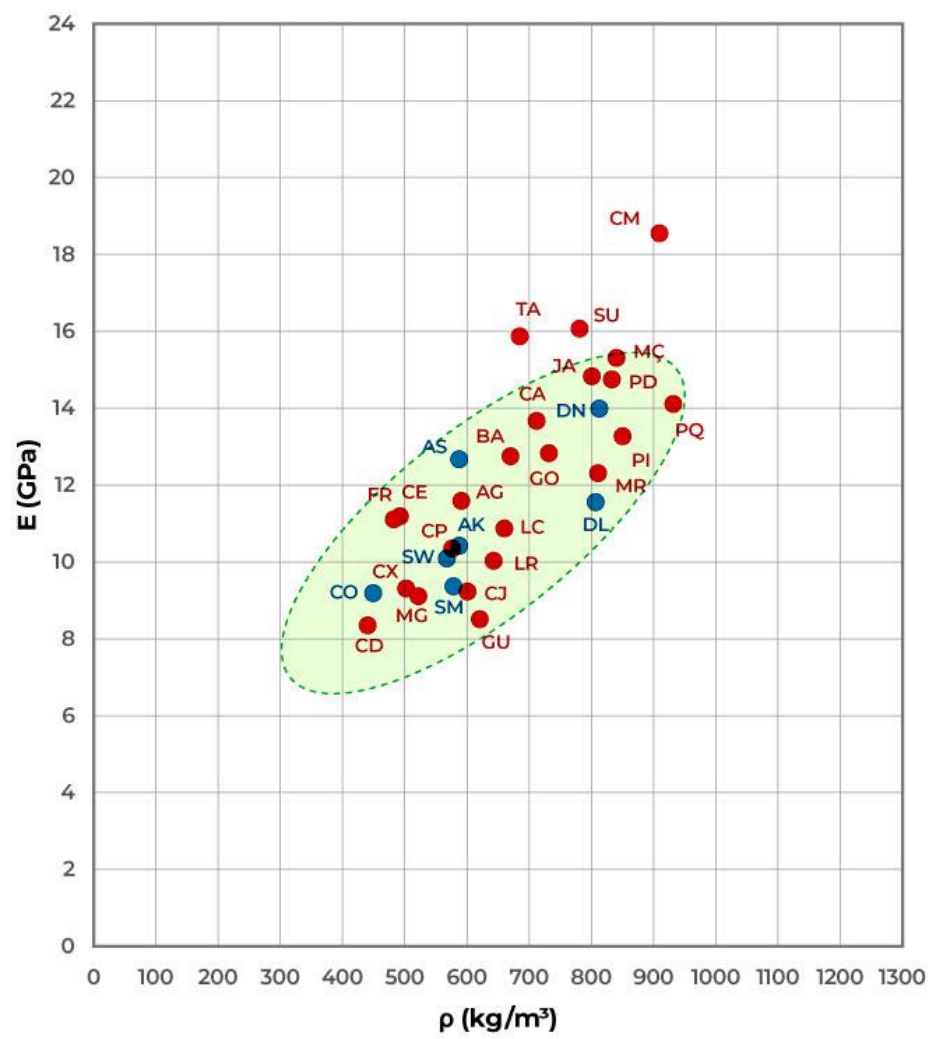

Figura 3: Gráfico de dispersão comparativo entre espécies tradicionalmente e comercializadas no Maranhão para o fundo e laterais do violão. Onde: círculos azuis - DA=Dalbergia nigra; $\mathrm{DL}=$ Dalbergia latifolia; $\mathrm{SW}=\mathrm{Swietenia}$ macrophylla; $\mathrm{SM}=$ Swietenia mahogani; $\mathrm{AK}=\mathrm{Acacia}$ koa; $\mathrm{CO}=\mathrm{Cordia}$ odorata; $\mathrm{AS}=\mathrm{Acer}$ saccharum; círculos vermelhos - Angelim=AG; Bacuri=BA; Cachaceiro=CA; Cedro=CD; Cedrorana $=\mathrm{CE}$; Cerejeira-da-Amazônia=CJ; Copaíba=CP; Cumaru=CM; Curupixá=CX; Freijó=FR; Goiabão=GO; Guanandi=GU; Jatobá=JA; Louro-cravo=LC; Louro-rosa=LR;

Maçaranduba=MÇ; Maracatiara=MR; Mogno=MG; Pau-d'arco=PD; Piqui=PI; Piquiá=PQ; Sucupira=SU e Tatajuba=TA. 
A partir da figura 3, é possível perceber a indicação destas madeiras nativas como materiais alternativos para o fundo e as laterais, alinhando-as às descrições já feitas. A literatura aponta a Dalbergia nigra (Jacarandá do Brasil) como a espécie mais utilizada para o fundo e as laterais do violão (TELES, 2004; YOSHIKAWA et al., 2008; YOSHIKAWA; WALTHAM, 2014; BENNETT, 2016).

Sendo assim, as relações de estreita proximidade são vistas entre a Copaíba e Acacia koa, Swietenia macrophylla e Swietenia mahogani. Do mesmo modo, a Cerejeira-da-Amazônia, Guanandi e Swietenia mahogani, o Jatobá, Maçaranduba, Pau-d'arco e Piqui em relação à Dalbergia nigra, bem como a Maracatiara e a Dalbergia latifolia. Outras madeiras analisadas encontram dentro do espectro delineado pelas madeiras "tradicionais", como o Angelim, Bacuri, Cachaceiro, Cedrorana, Curupixá, Freijó, Goiabão, Louro-cravo e Louro-rosa.

Ao observar-se a dureza e o quanto a madeira resiste até romper, existem significativas relações que contribuem para o estudo, com valores parecidos como: Cedrorana e Sucupira com Cedrela odorata, Swietenia mahogani e Swietenia macrophilla; Curupixá, Goiabão e Jatobá com Acacia koa; e Piqui e Dalbergia latifolia e Dalbergia nigra. Outras relações a serem consideradas, mesmo com uma certa distância, como: Bacuri e Dalbergia latifolia e Cachaceiro e Acer saccharum.

Diante do exposto para esta peça do violão, as espécies enquadradas nestes aspectos são: Angelim, Bacuri, Copaíba, Curupixá, Goiabão e Piquiá.

\section{Braço}

Stanciu, Curtu e Apostol (2014) falam que o braço do violão, mesmo em situação de repouso, onde o instrumentista não toca, está sujeito a cargas promovidas pelas cordas tensionadas, cordas estas com suas extremidades ligadas do corpo a esta peça. Esta parte do violão também é, conforme Zaczéski et al. (2018), responsável por sustentar a escala, parte na qual os dedos do violonista pressionam as cordas. O braço, por sofrer estes esforços externos, exige uma madeira que possua relativa resistência mecânica. Portanto, os bons índices de estabilidades dimensional e higroscópica, assim como a elevada dureza, correspondem aos requisitos que as madeiras desta peça devem possuir (GORE, 2011; SPROßMANN; ZAUER; WAGENFÜHR, 2017).

É possível ver na figura 4 como as referências das espécies-padrão são cada vez mais densas e elásticas para o braço, em relação ao tampo, fundo e laterais. Assim, também percebese as semelhanças entre espécies, com ênfase na relação entre o Angelim e a Juglans nigra, Cerejeira-da-Amazônia e Swietenia mahogani, e a Copaíba e a Swietenia macrophylla e Swietenia mahogani. Com menor intensidade, mas ainda próximos, veem-se: Louro-cravo e Acer saccharum e Juglans nigra, Goiabão e Pterocarpus soyauxii. Ademais, o espectro delimitado 
pelas madeiras "tradicionais" permite adequação de praticamente todas as espécies estudadas, porém a facilidade de acabamento e manejo durante a fabricação devem ser considerados.

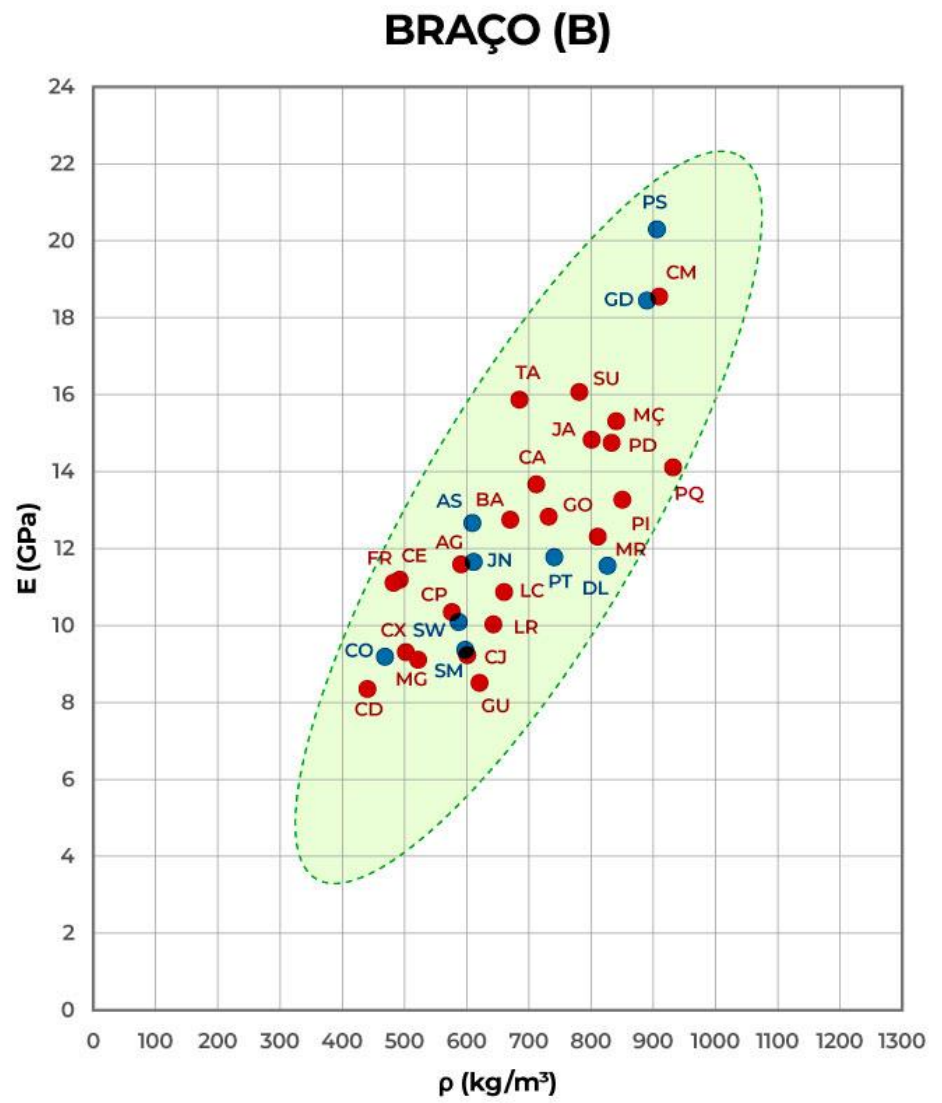

Figura 4: Gráfico de dispersão comparativo entre espécies tradicionalmente usadas e comercializadas no Maranhão para o braço do violão. Onde: círculos azuis - $\mathrm{DA}=$ Dalbergia nigra; $\mathrm{SW}=$ Swietenia macrophylla; $\mathrm{AS}=\mathrm{Acer}$ saccharum; $\mathrm{SM}=$ Swietenia mahogani; $\mathrm{CO}=$ Cordia odorata; $\mathrm{PS}=$ Pterocarpus soyauxii; $\mathrm{PE}=\mathrm{Peltogyne}$ spp; JN=Juglans nigra; GD=Guibourtia demeusei; círculos vermelhos - Angelim=AG; Bacuri=BA; Cachaceiro=CA; Cedro=CD; Cedrorana=CE; Cerejeira-da-Amazônia=CJ; Copaíba=CP; Cumaru=CM; Curupixá=CX; Freijó=FR; Goiabão=GO; Guanandi=GU; Jatobá=JA; Louro-cravo=LC; Louro-rosa=LR; Maçaranduba=MÇ; Maracatiara=MR; Mogno=MG; Pau-d'arco=PD; Piqui=PI; Piquiá=PQ; Sucupira=SU e Tatajuba=TA.

Destarte, outras propriedades mecânicas como o módulo de ruptura $(\mathrm{G})$ e a dureza Janka (Hj) refletem as seguintes relações de proximidade de valores entre espécies nativas e tradicionalmente usadas: Cachaceiro com Acer saccharum; Cedrorana e Sucupira com Cedrela odorata, Swietenia mahogani e Swietenia macrophilla; Louro-rosa com Piceas sitchensis e Guibourtia demeusei; e Maçaranduba com Juglans nigra. Pode-se dizer ainda que as demais madeiras estão dentro do espectro delimitado pelos valores das madeiras mundialmente usadas.

Existem também outras afinidades que cabem serem relatadas, ainda que com uma certa distância, como: Angelim com Acer saccharum; Bacuri com Pterocarpus soyauxii; Curupixá e Goiabão com Juglans nigra; Piqui com Dalbergia latifolia; Piquiá com Swietenia mahogani e Swietenia macrophilla. 
Conforme o avaliado, as espécies adequadas para a produção do braço do violão, conforme nível de anisotropia, trabalhabilidade e tipo de grã, são: Angelim, Bacuri, Copaíba, Curupixá, Goiabão e Piquiá.

\section{Escala}

Para Zaczéski et al. (2018), a escala é uma faixa de madeira dura e densa acoplada ao braço, onde nela o musicista faz a digitação das notas que são delimitadas por filetes metálicos, chamados de trastes. Como características necessárias para as madeiras da escala, Gore (2011) apresenta a resistência ao desgaste e estabilidade com variação da umidade. Diante disso, as escalas devem ter grande estabilidade dimensional, ter alta dureza e rigidez compressiva, além de serem acopladas a um braço estável (GORE, 2011).

Viu-se também que conforme à medida em que se avança sobre o estudo das peças do violão, mais se é exigido das madeiras em termos de elasticidade, dureza e resistência mecânica. Logo, percebe-se a restrição de espécies regionais que se ajustam ao perfil desejado para esta peça.

\section{ESCALA (E)}

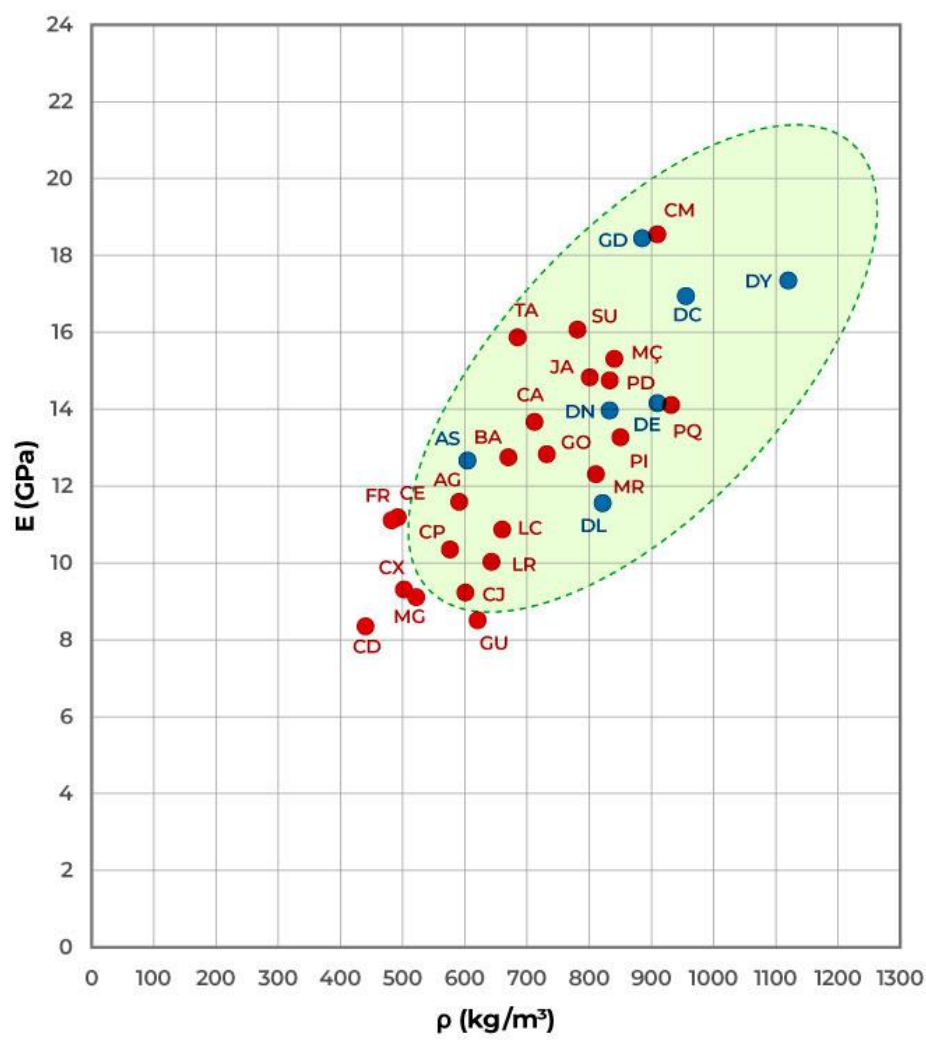

Figura 5: Gráfico de dispersão comparativo entre espécies tradicionalmente usadas e comercializadas no Maranhão para a escala do violão. Onde: círculos azuis - $\mathrm{DA}=$ Dalbergia nigra; $\mathrm{DL}=\mathrm{Dalbergia}$ latifolia; $\mathrm{DE}=\mathrm{Diospyros}$ ebenum; $\mathrm{AS}=\mathrm{Acer}$ saccharum; DC=Diospyros crassiflora; DY=Diospyros celebica; $G D=G u i b o u r t i a$ demeusei; círculos vermelhos Angelim=AG; Bacuri=BA; Cachaceiro=CA; Cedro=CD; Cedrorana=CE; Cerejeira-da-Amazônia=CJ; Copaíba=CP; Cumaru=CM; Curupixá=CX; Freijó=FR; Goiabão=GO; Guanandi=GU; Jatobá=JA; Louro-cravo=LC; Louro-rosa=LR; Maçaranduba=MÇ; Maracatiara=MR; Mogno=MG; Pau-d'arco=PD; Piqui=PI; Piquiá=PQ; Sucupira=SU e Tatajuba=TA 
A figura 5 aponta similaridades entre madeiras regionais e "tradicionais" que são vistas em algumas relações, como: Piquiá e Diospyros ebenum; Maracatiara e Dalbergia latifolia; e Jatobá, Maçaranduba e Pau-d'arco e Dalbergia Nigra.

Também são vistos para esta parte do violão os níveis de dureza Janka $(\mathrm{Hj})$ e módulo de ruptura $(G)$ das madeiras locais, se comparadas com espécies mundialmente usadas. Com isso, é possível observar a semelhança entre materiais, como: Angelim e Bacuri com Acer saccharum. Com menor proximidade e considerável relevância: Piqui e Maracatiara com Dalbergia latifolia, Dalbergia Nigra e Diospyros ebenum; e Cumaru com Diospyros ebenum e Guibourtia demeusei. As demais madeiras maranhenses encontram-se com valores até $40 \%$ menores que o previsto para esta peça do violão, o que demonstra maior fragilidade. Mesmo com tudo isso, o espectro formado pelas madeiras mundiais torna a maioria das espécies avaliadas aptas para a fabricação de escalas, exceto o Cedrorana, Curupixá, Freijó e Guanandi.

Por fim, ao considerar-se os fatores como trabalhabilidade, nível de anisotropia e tipo de grã, apenas o Angelim, Maçaranduba, Maracatiara e Piquiá encontram-se adequados para a fabricação de escala.

\section{Cavalete}

A ponte ou cavalete é uma peça de madeira colada sobre o tampo responsável pela transmissão das vibrações destas para o interior do corpo acústico e sustentá-las (WEGST, 2006; GORE, 2011; ZACZÉSKI et al., 2018). Em decorrência das tensões pelas quais é submetida, a ponte exige das madeiras características como alta rigidez e densidade (BENNETT, 2016), vistas em ébanos e jacarandás, como explicam Ncube e Masilinga (2017).

Diante de características como estas, esta parte do violão é, dentre as 5 estudadas, a que demanda maior resistência e elasticidade da madeira. Isso é nítido na figura 6 , que delineia a presença seletiva de madeiras nativas enquadradas para a fabricação da ponte, como o Piquiá (que se equipara ao Diospyros ebenum). Há outras espécies locais com certa proximidade que devem ser consideradas (apesar dos valores estarem um pouco abaixo), tais como Jatobá, Maçaranduba e Pau-d'arco, que se aproximam da Dalbergia nigra, e no mais abaixo ainda veem-se o Goiabão, Maracatiara e o Piqui. Dentro da análise de valores mecânicos já descritos das espécies, percebese que apenas o Louro-rosa e o Piqui assemelham-se com as madeiras "tradicionais" (Diospyros celebica). 


\section{CAVALETE (C)}

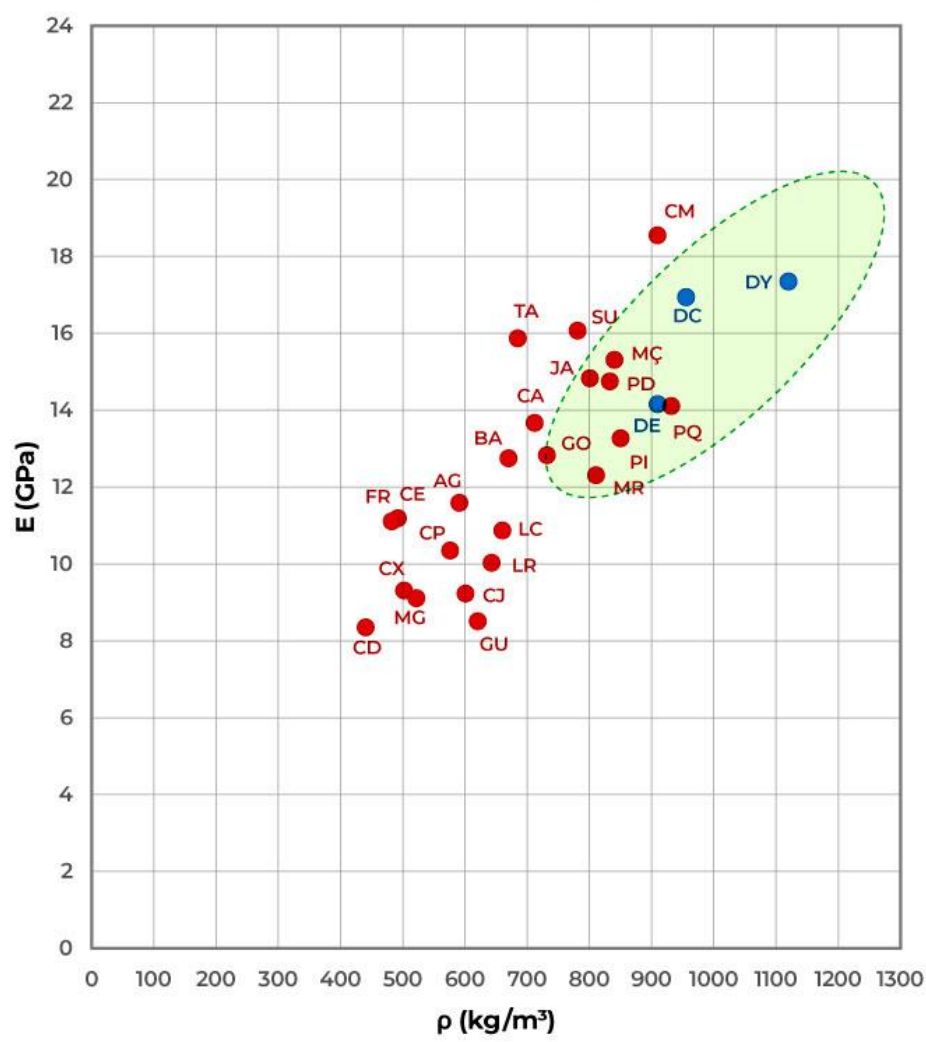

Figura 6: Gráfico de dispersão comparativo entre espécies tradicionalmente usadas e comercializadas no Maranhão para o cavalete do violão. Onde: círculos azuis - DE=Diospyros ebenum; DC=Diospyros crassiflora; DY=Diospyros celebica; círculos vermelhos - Angelim=AG; Bacuri=BA; Cachaceiro=CA; Cedro=CD; Cedrorana=CE; Cerejeira-da-Amazônia=CJ;

Copaíba=CP; Cumaru=CM; Curupixá=CX; Freijó=FR; Goiabão=GO; Guanandi=GU; Jatobá=JA; Louro-cravo=LC; Louro-rosa=LR; Maçaranduba=MÇ; Maracatiara=MR; Mogno=MG; Pau-d'arco=PD; Piqui=PI; Piquiá=PQ; Sucupira=SU e Tatajuba $=$ TA.

Trazendo as variáveis já trabalhadas para o desfecho da avaliação desta parte de violão (trabalhabilidade, coeficiente de anisotropia e tipo de grã), as madeiras consideradas aptas são: a Maçaranduba e o Piquiá.

\section{Considerações Finais}

Com os gráficos de dispersão da densidade e módulo de elasticidade elaborados para esta pesquisa, bem como o apoio de algumas propriedades mecânicas (módulo de ruptura e dureza Janka) e outras variáveis (tipo de grã, coeficiente de anisotropia e nível de trabalhabilidade), foi possível concluir que nove espécies maranhenses apresentaram resultados satisfatórios para aplicação em peças do violão (Angelim, Bacuri, Copaíba, Curupixá, Goiabão, Maçaranduba, Maracatiara e Piquiá). Sendo assim, as madeiras aqui listadas seguem, como critério, o tipo de peça (tampo, fundo e laterais, braço, escala e cavalete) e nível de qualidade (adequadas).

Para tampos, as madeiras adequadas são: Bacuri, Curupixá e Goiabão. Para a construção de fundos e laterais, sugerem-se: Angelim, Bacuri, Copaíba, Curupixá, Goiabão e Piquiá. As 
madeiras para braços, ainda que todas as espécies deste artigo estejam dentro do espectro aceitável, são: Angelim, Bacuri, Copaíba, Curupixá, Goiabão e Piquiá. Para escalas, indicam-se: Angelim, Maçaranduba, Maracatiara e Piquiá. E para a confecção de cavaletes estão: Maçaranduba e o Piquiá.

De certo, seis madeiras locais podem de forma razoável serem usadas, dependendo do tipo de qualidade que se deseja para o instrumento, se não forem considerados o coeficiente de anisotropia e tipo de grã, uma vez que a lista de espécies usadas internacionalmente que ultrapassam o índice de 2,0 (Piceas abies - 2,10, Albies albes - 2,18, Dalbergia latifolia - 2,19 e Acer saccharum - 2,06). Portanto, madeiras como Cedrorana, Cerejeira-de-Amazônia, Freijó, Guanandi, Jatobá, Louro-rosa e Piqui, mesmo com valor acima de 2,0 podem ser razoavelmente utilizadas, uma vez que são de grã regular e fácil trabalhabilidade. Pautado nisso, Também é possível fabricar tampos, fundos e laterais e braços com a Cedrorana, Freijó, Guanandi, Jatobá e Piqui. A Cerejeira-de-Amazônia pode ser aplicada para fundos e laterais, bem como braços de violão. Já para confeccionar escalas, apenas o Jatobá e Piqui. E, finalmente, apenas o Piqui serve para o fabrico de cavaletes.

Por fim, por se tratar de um estudo pautado em cruzamento de informações retiradas da literatura, sugere-se a realização de ensaios de ordem acústica, como também a fabricação de um violão experimental e testes de usabilidade, de modo a analisar os dados levantados neste trabalho.

\section{Referências}

ALVES, E.S.; LONGUI, E.L.; AMANO, E. Pernambuco wood (Caesalpinia echinata) used in the manufacture of bows for string instruments. IAWA Journal, Vol. 29 (3), 2008. Disponível em: https://www.researchgate.net/publication/266218806_Pernambuco_wood_Caesalpinia_echi nata used in the manufacture of bows for string instruments. Acesso em: 15.out.2018.

ANDRADE, M. Pequena história da música. Rio de Janeiro: Nova Fronteira, 2015.

ARAMAKI, M.; BRANCHERIAU, H. B. L.; YSTAD, R. K. M. S. Sound quality assessment of wood for xylophone bars. Journal of Acoustical Society of America. 121 (4). 2007. p. 2407-2420. Disponível em: http://kronland.fr/wpcontent/uploads/2016/08/jasa_Xylophone.pdf. Acesso em: 18.jun.2018.

BENNETT, B. C.; The Sound of Trees: Wood Selection in Guitars and Other Chordophones. Economic Botany, $\mathrm{XX}(\mathrm{X})$. New York: 2016. p. 1-15. Disponível em: https://link.springer.com/article/10.1007/s12231-016-9336-0. Acesso em: 19.jun.2018.

BESSA, F. M. S. Caracterização Anatómica, Física, Química e Acústica de Madeiras de Várias Espécies para a Construção de Instrumentos Musicais - Uma Aplicação à Viola Dedilhada. Dissertação (Mestrado em Engenharia dos Materiais Lenhocelulósicos). Instituto Superior de Agronomia. Universidade Técnica de Lisboa, Lisboa: 2000. Disponível em: http://www.ipef.br/servicos/teses/arquivos/bessa,fms-m.pdf. Acesso em: 17.jun.2018. 
BRÉMAUD, I. Acoustical properties of wood in string instruments soundboards and tuned idiophones: Biological and cultural diversity. Journal of Acoustical Society of America. 131 (1). 2012. p. 808-818. Disponível em: https://hal.archives-ouvertes.fr/hal00808347/document. Acesso em: 19.jun.2018.

BRÉMAUD, I.; CABROLIER, P.; MINATO, K.; GÉRARD, J.; THIBAUT, B. Vibrational properties of tropical woods with historical uses in musical instruments. In: ESWM CONFERENCE WOOD SCIENCE FOR THE PRESERVATION OF CULTURAL HERITAGE, 2008, Braga. Proceedings e Report, 68. Braga: ESWM, 2008. 1 CD-ROM. Disponível em: https://www.torrossa.com/en/resources/an/2443364\# Acesso em: 19.jun.2018

BUCUR, V. Acoustics of wood. The Thirteenth International Congress on Sound and Vibration (ICSV13). Vienna: 2006. Disponível em: http://blogs.umass.edu/bct597fclouston/files/2011/01/acoustics-of-wood.pdf. Acesso em: 21.ago.2018

CHIARELLI, L. K. M. A música como meio de desenvolver a inteligência e a integração do ser. Revista Recre@ rte n.3 Junho. Instituto Catarinense de Pós-Graduação, 2005,3:1-10. Disponível

em: https://www.researchgate.net/publication/266446626_A_MUSICA_COMO_MEIO_DE_D ESENVOLVER A INTELIGENCIA E A INTEGRACAO DO SER. Acesso em: 15.dez.2018.

CLAYTON, M.; HERBERT, T.; MIDDLETON, R. The cultural study of music: a critical introduction. Taylon \& Francis Books Inc., New York, 2003. p. 370. Disponível em: https://www.posgrado.unam.mx/musica/lecturas/musicologia/complementarias/nuevaMusic ologia/Clayton\%20et\%20al_The\%20Cultural\%20Study\%20of\%20Music\%202003.pdf.

Acesso em: 15.set.2018.

COSTA, J. A. Classificação de madeiras da Amazônia para composição de instrumento musical de corda através da técnica de excitação por impulso. Dissertação (Mestrado em Ciências Florestais e Ambientais). Universidade Federal do Amazonas, Manaus, 2017. Disponível em: https://tede.ufam.edu.br/bitstream/tede/5649/5/Disserta\%C3\%A7\%C3\%A3o\%20\%20Joelma\%20A.\%20Costa.pdf. Acesso em: 25.mai.2018

FLORES, A. L. Análise de diferentes madeiras brasileiras em substituição às espécies tradicionais no violão clássico. Dissertação (Mestrado em Engenharia e Ciência dos Materiais). Programa de Pós-Graduação em Engenharia e Ciência dos Materiais - PIPE. Setor de Tecnologia. Universidade Federal do Paraná, Curitiba,2015. Disponível em: http://www.pipe.ufpr.br/portal/defesas/dissertacao/295.pdf. Acesso em: 25.mai.2018.

GORE, T. Proceedings of Meetings on Acoustics. 161st Meeting Acoustical Society of America. vol 12. 035001. Seattle, Washington. Session 3Amu, Musical Acoustics. 2011. Disponível em: http://www.goreguitars.com.au/attachments/POMA_paper.pdf. Acesso em: 26.jun.2018.

KÚDELA, J.; KUNŠTÁR, M. Physical-acoustical characteristics of maple wood with wavy structure. Annals of Warsaw University of Life Sciences - SGGW. Forestry and Wood Technology. No 75, 2011: p 12-188. Disponível em: http://annalswuls.sggw.pl/files/files/fwt/fwt2011no75art02.pdf. Acesso em: 30.mai.2018.

LONGUI, E. L. Potencial de madeiras nativas na fabricação de arcos para instrumentos de corda. Tese (Doutorado em Biodiversidade vegetal e Meio Ambiente). Instituto de 
Botânica da Secretaria do Meio Ambiente, São Paulo, 2009. Disponível em: http://www.scielo.br/pdf/\%0D/abb/v19n4/a18v19n4.pdf. Acesso em: 26.mai.2018.

LONGUI, E. L.; LOMBARDI, D. R.; ALVES, E. S. Six potential woods for bows of stringed instruments: organoleptic properties, machining and commercial availability. Rev. Inst. Flor. v. 23 n. 2 p. 203-216, 2011. Disponível em: https://www.researchgate.net/publication/272166117_Six_potencial_woods_for_bows_of_s tringed_instruments_organoleptic_properties_machining_and_commercial_availability_Sei s_madeiras_potenciais_para_arcos_de_instrumentos_de_corda_propriedades_organolepti. Acesso em: 27.mai.2018.

MORESCHI, J. C. Propriedades tecnológicas da madeira. Departamento de Engenharia e Tecnologia Florestal, Universidade Federal do Paraná, Curitiba, 2009.

NAHUZ, M. A. R.; MIRANDA, M. J. A. C.; IELO, P. K. Y.; PIGOZZO, R. J. B.; YOJO, T. Catálogo de madeiras brasileiras para a construção civil. São Paulo: IPT - Instituto de Pesquisas Tecnológicas do Estado de São Paulo, 2013. Disponível em: https://www.ipt.br/download.php?filename $=980-$

Catalogo_de_Madeiras_Brasileiras_para_a_Construcao_Civil.PDF. Acesso em: 15.mai.2018.

NCUBE, E.; MASILINGA, P. Prospective Zambian tonewoods for dreadnought acoustic guitar. International Wood Products Journal, vol 8, no 4, 2017. p 216-226. Disponível em: https://www.tandfonline.com/doi/full/10.1080/20426445.2017.1391964. Acesso em: 11.mai.2018.

OLIVEIRA, J. T. S.; DELLA LUCIA, R. M.; VITAL, B. R. Estudo das propriedades físicas e tecnológicas da madeira da pindaíba (Xylopia sericea St. Hil.). Revista Árvore, v.12, n.2, p. 129-133, 1988.

PORTELA, M. S. Estudo das propriedades acústicas da madeira amazônica Marupá para tampo de violão. Tese (Doutorado em Engenharia Mecânica). Programa de PósGraduação em Engenharia Mecânica. Universidade Federal de Santa Catarina, Florianópolis, 2014. Disponível em: https://repositorio.ufsc.br/bitstream/handle/123456789/132450/332924.pdf?sequence=1\&is Allowed=y. Acesso em: 16.mai.2018.

SOUZA, M. R. de. Classificação de madeiras para instrumentos musicais. Brasília: IBDF / DEL / LPF, 1983. 21p.

SLOOTEN, H. J.; SOUZA, M. R. Avaliação das espécies madeireiras da Amazônia selecionadas para a manufatura de instrumentos musicais. INPA, Manaus, 1993. 141 p.

SOARES, M. A. Produção de um violão clássico em madeira de Teca (Tecnota grandis). Trabalho de Conclusão de Curso (Graduação) Curso de Engenharia Industrial Madeireira. Universidade Estadual Paulista, São Paulo, 2014. Disponível em: https://repositorio.unesp.br/bitstream/handle/11449/142904/000867439.pdf?sequence=1. Acesso em: 27.mai.2018.

SPROßMANN, R.; ZAUER, M.; WAGENFÜHR, A. Characterization of acoustic and mechanical properties of common tropical woods used in classical guitars. Results in Physics, v. $7 . \quad$ p. 1737-1742. $2017 . \quad$ Disponível em: https://www.researchgate.net/publication/316873645 Characterization of acoustic and m echanical properties of common tropical woods used in classical guitars. Acesso em: 14.mai.2018. 
STANCIU, M. D.; CURTU, I.; APOSTOL, D. Evaluation of strain and stresses states of reinforced guitar neck with finite elements method. 3rd International Conference "Research \& Innovation in Engineering" COMAT 2014 16-17 October 2014, Braşov, Romania. Disponível em: http://aspeckt.unitbv.ro/jspui/bitstream/123456789/645/1/31 stanciu n 167-172.pdf. Acesso em: 16.set.2018.

TELES, R. F. Influence of wood finishing products on the vibro-acoustical properties of tropical woods. 23rd International Congress on Sound and Vibration. Athens, 2016.

VASCONCELlOS, F. J.; FREITAS, J. A.; LIMA, V. M.O. C.; MONTEIRO, L. V.; PEREIRA, S. J. Madeiras Tropicais de Uso Industrial no Maranhão: características tecnológicas. Instituto Nacional de Pesquisas da Amazônia (INPA), Universidade Federal do Maranhão (UFMA), Manaus, 2001. 96 p.

WEGST, U. G. K. Wood for sound. American Journal of Botany 93(10). 1439-1448. 2006. Disponível em: https://onlinelibrary.wiley.com/doi/epdf/10.3732/ajb.93.10.1439. Acesso em: 10.mai.2018.

YOSHIKAWA, S.; SHINODUKA, M.; SENDA, T. A comparison of string instruments based on wood properties: Biwa vs. cello. Acoustical Science and Technology, 29, 1. Japan, 2008. Disponível em: https://www.jstage.jst.go.jp/article/ast/29/1/29_1_41/_pdf/-char/en. Acesso em: 12.mai.2018.

YOSHIKAWA, S.; WALTHAM, C. Woods for Wooden Musical Instruments. ISMA 2014, Le Mans, 2014. Disponível em: http://www.conforg.fr/isma2014/cdrom/data/articles/000038.pdf. Acesso em: 13.mai.2018.

ZACZÉSKI, M.; BECKER, C. H.; BARROS, T. G.; FERREIRA, A. L.; FREITAS, T.C.; Violão: aspectos acústicos, estruturais e históricos. Revista Brasileira de Ensino de Física, vol. 40, no 1, e1309. 2018. Disponível em: http://www.scielo.br/pdf/rbef/v40n1/1806-1117-rbef40-01-e1309.pdf. Acesso em: 14.jun.2018.

\section{Sobre o autor}

\section{David Guilhon}

Bacharel em Desenho Industrial (UFMA) e Mestre em Design (UFMA). Professor e pesquisador do curso de Design (Universidade Ceuma). CEO da Boomerang Store. Atua em pesquisas sobre design de instrumentos musicais, madeiras brasileiras (e maranhenses) e processos produtivos, bem como processos de produção gráfica digital (sublimação).

ORCID 0000-0002-1215-6876.

\section{Karoline de Lourdes Monteiro Guimarães}

Tecnóloga em Design de Interiores (Universidade CEUMA), Bacharel em Desenho Industrial (UFMA) e Mestre em Design (UFMA). Professor e pesquisador dos cursos de Design e Arquitetura e Urbanismo (Universidade Ceuma). Atua em pesquisas sobre design de instrumentos musicais, madeiras brasileiras (e maranhenses) e processos produtivos, bem como design de produto e de interiores.

ORCID 0000-0002-9193-6247.

\section{Anna Karen Lima Lourenço}

Graduanda de Design (Universidade CEUMA). Atualmente é aluna-pesquisadora de PIBIC na área de design de instrumentos musicais. Tem interesse em design gráfico e de produtos sustentáveis.

ORCID 0000-0003-3262-4216. 